\title{
National Vulnerability Model based on Principal Component Analysis
}

\author{
Ziming Liu \\ School of North China Electric Power University, Baoding 071000, China. \\ 932899937@qq.com
}

Keywords: Principal component analysis; Climate change; National vulnerability; Fragile States Index.

Abstract: In order to analyze the impact of climate change on national stability, a model named improved principal component analysis is established to measure the fragility of a state.

We use the Seven climate-fragility risks that G7 has raised as our indicators , including Local resource competition, Livelihood insecurity and migration ,Extreme weather events and disasters ,volatile food prices and provision ,Transboundary water management, Sea level rise and coastal degradation and Unitended effects of climate policies. We subdivide these seven indicators into 18 influencing factors.We use the improved principal component analysis as our basic model for the evaluation of national fragility. We introduce a new variable SF as a comprehensive score for a country's vulnerability. Through the eigenvector, we can get the main factors that affect the fragility. For the sake of verifying our model. We have chosen eight countries with greatly different vulnerabilities to rank and compare with the ranking of the national fragility given by Fragile States Index, and then come to a consistent conclusion.

\section{Introduction}

\subsection{Background}

Climate change is one of the most pervasive and threatening issues of our time, with far-reaching impacts in the twenty-first century[1].Today, the negative impacts of climate change are on the rise, and issues such as droughts, melting glaciers and sea-level rise are slowly changing the way people live.

Not only that, the harm caused by climate change, such as natural disasters; the reduction of arable land; the unstable weather and the rising temperature, will even endanger the stability of a country and exacerbate the already fragile state. Even causing violent conflicts and other unpredictable hazards.

Therefore, it is particularly important to study the impact of climate on the fragility of a state. 


\subsection{Variable Description}

We will show the description of variables in Table 1.

Table 1: Variable Description

\begin{tabular}{c|c}
\hline Comprehensive score of state's fragility & $\mathrm{SF}$ \\
\hline Level of state's fragility & $\mathrm{SFL}$ \\
\hline Predict score & $\mathrm{PS}$ \\
\hline Total supply of water & $\mathrm{T}$ \\
\hline Renewable water & $\mathrm{RW}$ \\
\hline Agricultural land & $\mathrm{Al}$ \\
\hline Irrigated area & $\mathrm{Ia}$ \\
\hline Forest coverage & $\mathrm{FC}$ \\
\hline Unemployment rate & $\mathrm{Ur}$ \\
\hline Fishery industry & $\mathrm{Fi}$ \\
\hline GNI & $\mathrm{GNI}$ \\
\hline Number of immigrants & $\mathrm{Noi}$ \\
\hline Total population & $\mathrm{Tp}$ \\
\hline Consumer price & $\mathrm{D}$ \\
\hline Agricultural output & $\mathrm{Cp}$ \\
\hline Disaster risk reduction progress score & $\mathrm{Ao}$ \\
\hline Sea level height & $\mathrm{P}$ \\
\hline Air pollution & $\mathrm{S}$ \\
\hline Greenhouse gases & $\mathrm{Ap}$ \\
\hline Diversity of fish & $\mathrm{Ga}$ \\
\hline & $\mathrm{Df}$ \\
\hline
\end{tabular}

\section{State's Fragility Metric Model}

\subsection{Develop our metric model}

\subsubsection{Analyze the indicators}

After consulting a lot of literature, we found that it is necessary to combine climate change with 12 indicators given by Fragile States Index [2] to analyze the fragility of a state.

In order to link this combination to state's fragility when the impacts of cli- mate change interact with other stress, we decided to use the seven compound climate-interacts risks proposed by the G7 member countries [3] as our evaluation indicators.

The seven compound climate-interacts risks are:

Resource competition (Lr): As the pressure on natural resources increases, competition can lead to instability and even violent conflict in the absence of effective dispute resolution.

Livelihood Insecurity and migration (Li): Climate changes will increase the human insecurity of people who depend on natural resources for their livelihoods, which could push them to migrate or to turn to informal and illegal sources of income.

Extreme weather events and disasters (Ew): Extreme weather events and disasters will exacerbate fragile situations and can increase people's vulnerability and grievances, especially in countries affected by conflict. 
Volatile food prices and provision (Vf): Climate change is highly likely to disrupt food production in many regions, increasing prices and market volatility, and heightening the risk of protests, rioting, and civil conflict.

Transboundary water management (Tw): Transboundary water management is frequently a source of tension. As demand grows and climate impacts affect availability and quality, competition over water use will likely increase pressure on existing governance structures.

Sea level rise and coastal degradation (Sr): Rising sea levels will threaten the viability of low lying areas even before they are submerged, leading to social disruption, displacement, and migration.At the same time, disagreements over maritime boundaries and ocean resources may increase.

Unintended effects of climate policies (Ue): As climate adaptation and mitigation policies are more broadly implemented, the risks of unintended negative effects-particularly in fragile contexts-will also increase.

\subsubsection{Model's establishment}

After setting up the evaluation indicators, in order to facilitate our calcula- tion and evaluation, we looked up the factors that affect the climate, and then refered to the A New Climate for Peace[3], and we have made the corresponding concretion of the risk of seven kinds of composite climate vulnerability.As shown in the following Table 2:

Table 2: The corresponding concretion of indicators

\begin{tabular}{|c|c|c|}
\hline $\mathrm{Lr}$ & $\begin{array}{c}\text { water } \\
\text { land } \\
\text { timber }\end{array}$ & $\begin{array}{c}\text { Total supply of water(billion cubic meters) Renewable } \\
\text { water(billion cubic meters) Agricultural land(sq. km) } \\
\text { Irrigated area Forest coverage(sq. km) }\end{array}$ \\
\hline $\mathrm{Li}$ & $\begin{array}{c}\text { livelihood Insecurity } \\
\text { migration }\end{array}$ & $\begin{array}{c}\text { Unemployment rate(\% of total labor force) Fishery } \\
\text { industry(metric tons) } \\
\text { GNI(constant LCU) }\end{array}$ \\
\hline $\mathrm{Ew}$ & disasters & Disaster risk reduction progress score(1-5scale) \\
\hline $\mathrm{Vf}$ & & $\begin{array}{c}\text { Consumer price (annual \%) } \\
\text { Agricultural output(kt) }\end{array}$ \\
\hline $\mathrm{Tw}$ & & Per capita water consumption \\
\hline $\mathrm{Sr}$ & & Sea level height \\
\hline $\mathrm{Ue}$ & risk & Air pollution(micrograms per cubic meter) Greenhouse \\
gases(kt) & Diversity of fish \\
\hline
\end{tabular}

Through our analysis, we can get a matrix about the factors that we specify of a country in recent years. Because there are too many factors to be considered, so we decided to achieve dimensionality reduction by principal component analysis, and get $\mathrm{m}$ strongest correlation factors(principal component).Finally, we can get a formula:

$$
S F=\sum_{i=1}^{m} \alpha_{i} F_{i} \quad i=1,2, \cdots m
$$

Among it,SF is our newly defined variable, which represents the comprehensive score of state fragile. The higher the comprehensive score, the smaller the country's fragility.ai represents the contribution rate of the variance (information) of a certain principal component,Fi shows the region's score on state fragility at a certain year.With this calculation, the comprehensive score we get SF can determine the fragility of a country and measure the impact of climate change. 


\subsubsection{The process of establish our model}

After the variable setting is completed, we can get a matrix as follows:

$$
\left(\begin{array}{ccc}
x_{11} & \cdots & x_{1 j} \\
\vdots & \ddots & \vdots \\
x_{i 1} & \cdots & x_{i j}
\end{array}\right) \quad i=1,2,3 \cdots n ; j=1,2,3 \cdots t
$$

$x_{i j}$ represents the value of $x_{j}$ in the year of $\mathrm{i}$

we use Principal Component Analysis(PCA) to establish our model

But in actual calculation, the dimension of the index is often different.So the influence of dimension should be eliminated before the Principal Component Calculation.There are many ways to eliminate the dimension of data. The most common way is to standardize the original data, which is to do the following data transformation:

$$
\overline{x_{j}}=\frac{1}{n} \sum_{i=1}^{n} x_{i j} \quad s_{j}{ }^{2}=\frac{1}{n-1} \sum_{i=1}^{n}\left(x_{i j}-\overline{x_{j}}\right)^{2}
$$

When the random variable is normalized, its covariance is the same as the correlation coefficient.That is to say, the covariance matrix is its correlation coefficient matrix after standardization.

According to the formula of covariance, we can deduce that the covariance after standardized is the correlation coefficient of the original variable, that is to say, the covariance matrix of the standardized variables is the correlation matrix of the original variables. Furthermore, we can infer that whether standardization does or not, the correlation coefficient matrix of the variable will not be influenced.

So then we'll calculate the correlation coefficient matrix of the variable.

$$
\begin{array}{r}
\sum\left(s_{i j}\right)_{p \times p} \\
s_{i j}=\frac{1}{n-1} \sum_{i=1}^{n}\left(x_{k i}-\overline{x_{i}}\right)\left(x_{k j}-\overline{x_{j}}\right) \quad i, j=1,2, \cdots p
\end{array}
$$

After the covariance matrix is obtained, We can calculate its eigenvalue $\lambda_{i}$, We can calculate its eigenvalue and its Unit orthogonalized eigenvectors ai,Through the eigenvector, we can see the main factors affecting the state's fragility in this area. $\sum$ has $m$ larger eigenvalues $\lambda_{1} \geq \lambda_{2} \geq \cdots \lambda_{i}>0$, which are respectively the variance of the m principal components.Therefore, the ith principal component Fi of the original variable is:

$$
F_{i}=\alpha_{i}{ }^{\prime} X
$$

The variance (information) contribution rate of the principal component is used to reflect the amount of information, $\alpha \mathrm{i}$ is:

$$
\alpha_{i}=\lambda_{i} / \sum_{i=1}^{m} \lambda_{i}
$$

Then we need to select a few principal components F1, F2, $\cdots \cdots, F m, m$ is determined by the variance (information) cumulative contribution $\mathrm{G}(\mathrm{m})$ : 


$$
G(\mathrm{~m})=\sum_{i=1}^{m} \lambda_{i} / \sum_{k=1}^{p} x_{k}
$$

When the cumulative contribution rate is greater than $85 \%$, it is considered enough to reflect the information of the original variable, and the corresponding $\mathrm{m}$ is the extracted $\mathrm{m}$ principal components.

Next we can calculate the principal component load.The principal component load reflects the correlation between the principal component $\mathrm{Fi}$ and the original variable $\mathrm{Xj}$.

$$
l\left(Z_{i}, X_{j}\right)=\sqrt{\lambda_{i}} a_{i j} \quad(i=1,2, \cdots, m ; j=1,2, \cdots, p)
$$

After the principal component analysis, we can get the analysis result: The "component matrix" reflects the principal component load matrix. Then,we can calculate the score of the original data on the M principal component.

Finally, we can get the comprehensive score in the region:

$$
\begin{array}{r}
F_{i}=\alpha_{1 i} X_{1}+\alpha_{2 i} X_{2}+\cdots \alpha_{P i} X_{P} \quad \mathrm{i}=1,2, \cdots, \mathrm{m} \\
Z=\sum_{i=1}^{m} \alpha_{i} F_{i} \quad \mathrm{i}=1,2, \cdots, \mathrm{m}
\end{array}
$$

Based on our model, we can calculate the comprehensive score of acountry's national vulnerability for a particular year.

We can select several countries from all countries given by the Fragile States Index, rank the scores after using our model to calculate the composite scores, and then compare the results with the existed ranking comparison, if the rankings are consistent,we can prove the correctness of our model.

\section{Evaluation model}

First, we chose five more vulnerable countries, Congo Democratic Republic, Yemen,Iraq,Nigeria and Syria. We found that their scores were very similar and did not clearly show the difference. So we added three vulnerable countries like Namibia, Italy, and Australia. After we calculated them with the model, we got Figure 1 to show their scores. Table 3 shows the ranking given by Fragile States Index. We can see that the rankings obtained by our model are the same as the standard ones, so we can draw the conclusion that our model is correct.

Table 3: The standard Ranking

\begin{tabular}{c|c}
\hline State & Ranking \\
\hline congo Democratic Republic & 2nd \\
Yemen & 6 th \\
Iraq & 11 th \\
Nigeria & 16 th \\
Syria & 21 st \\
Namibia & 108 th \\
Italy & 147 th \\
Australia & 169 th
\end{tabular}




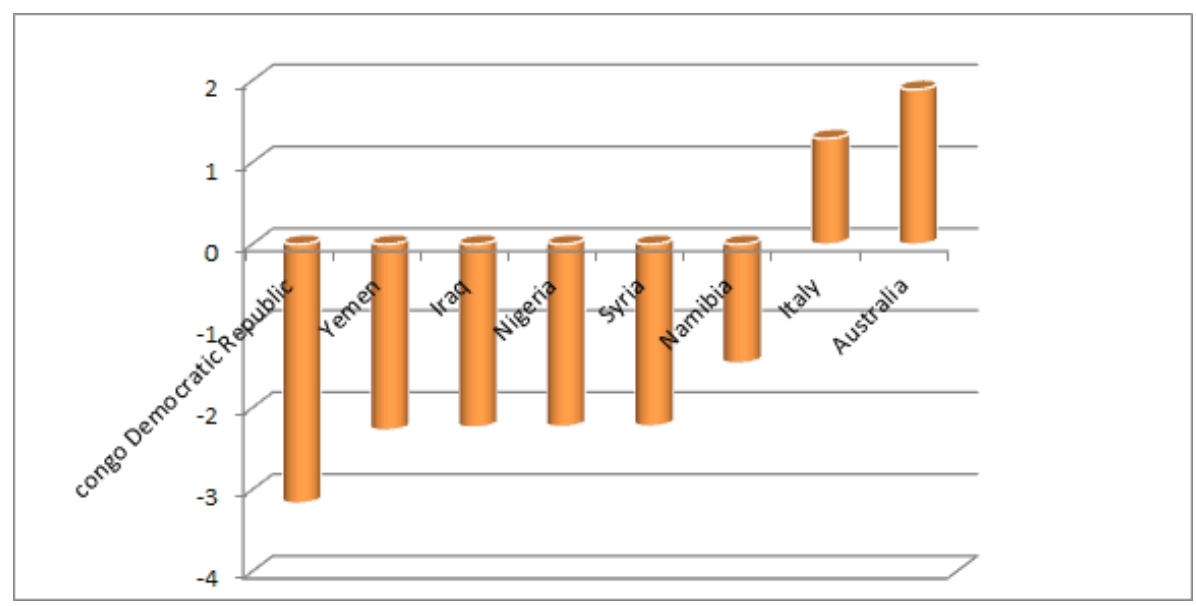

Figure 1: The scores of eight countries

\section{Strengths and Weaknesses}

\subsection{Strengths}

The indicators we have defined are comprehensive and authoritative. The seven composite climate-interacts risks can be directly linked to statefragility which can make the model more rational.

Our model is simple and universal. We can get a country's comprehensive score with simple data, and apply to most countries

Our model is realistic. We can use our model to predict the future fragility of a country.

\subsection{Weaknesses}

Most of the countries we choose are suffering and some of the data is incomplete, which may have some effect on the accuracy of the results.

Our model is unreliable when unidentified factors arise.

When we define the indicator, we may have some influential factors not considered.

\section{References}

[1] United Nations Environment Programme. Retrieved https://www.unenvironment.org/explore-topics/climate-change/why-does-climate-change-matter

[2] Fragile States Index. Retrieved from http://fundforpeace.org/fsi/ data/

[3] A New Climate for Peace: G7 Take Action on Climate and Fragility Risks. Retrieved from https://www.climate-diplomacy.org/the-initiative/new-climate-peace-g7-take-action-climate-and-fragility-risks 